\title{
Avaliação da qualidade dos índices de vegetação (NDRE e NDVI) para a cultura do amendoim, utilizando cartas de controle
}

Submetido - 01 ago. $2020 \quad$ Aprovado - 20 set. $2020 \quad$ Publicado - 14 out. 2020
dol http://dx.doi.org/10.17648/sas.v1i1.95

\section{Jarlyson Brunno Costa Souza}

Mestrando do Programa de Pós Graduação em Agronomia (Produção Vegetal) - Universidade Estadual Paulista "Julio de Mesquita Filho", Jaboticabal, São Paulo - Instituição, email:jarlyson.brunno@unesp.br.

Samira Luns Hatum de Almeida (D) Doutoranda do Programa de Pós Graduação em Agronomia (Produção Vegetal) - Universidade Estadual Paulista “Julio de Mesquita Filho”, Jaboticabal, São Paulo, e-mail:samiraluns@hotmai.com.

\section{Armando Lopes de Brito Filho (iD}

Mestrando do Programa de Pós Graduação em Agronomia (Ciência do solo) - Universidade Estadual Paulista "Julio de Mesquita Filho",Jaboticabal, São Paulo, e-mail:armandolopes9@hotmail.com.

\section{Franciele Morlin Carneiro iD}

Pós-Doutorando do Programa de Pós Graduação em Agronomia - Universidade Estadual Paulista “Julio de Mesquita Filho",Jaboticabal, São Paulo, e-mail: franmorlin1@gmail.com.

\section{Adão Felipe do Santos (iD}

Pós-Doutorando do Programa de Pós Graduação em Agronomia - Universidade Estadual Paulista “Julio de Mesquita Filho",Jaboticabal, São Paulo, e-mail:adaofeliped@gmail.com.

Prof. Dr. Livre Docente da Unesp/FCAV e-mail: rouverson@gmail.com.

\section{Rouverson Pereira da Silva}

\section{RESUMO}

O amendoim é considerado dentre as leguminosas uma das mais importantes, não só pela sua expressão econômica como também nutricionalmente. Técnicas de sensoriamento remoto aparecem como instrumento de elevado potencial, para auxiliar no desenvolvimento da cultura do amendoim. Diante disto, objetivou-se avaliar qual índice de vegetação possuem melhor qualidade para o monitoramento da cultura do amendoim. 0 experimento foi realizado em Fazenda comercial localizada em Dumont no Estado de São Paulo. 0 delineamento experimental foi baseado nas premissas do Controle Estatístico de Qualidade (CEQ) (Montgomery, 2009) contendo 30 pontos amostrais com malha $30 \times 30 \mathrm{~m}$. As avaliações foram realizadas durante o desenvolvimento da cultura aos 45, 65, 75 e 85 dias após a semeadura (DAS). Foram avaliados os índices de vegetação (IVs) NDVI e NDRE, com 0 auxílio dos sensores de dossel GreenSeeker e OptRX. A análise da variabilidade da reflectância e dos IVs da cultura do amendoim foi realizada por meio de cartas de controle de valores individuais. $O$ agrupamento dos dados observado aos 45 DAS, apresentou amplitude de variação maior no NDVI. Em 75 e 85 dias após a semeadura (DAS), o agrupamento dos valores para o NDVI pode ser constatado pela proximidade dos pontos em relação à média, na carta de valores individuais, ou seja, houve baixa variabilidade da aquisição dos dados. Foi observado que o índice NDRE apresentou maior qualidade do processo por ter menor variabilidade. Outro fator constatado, foi que o reflectância do solo não influenciou não qualidade do processo como aconteceu com o NDVI, que teve uma alta variabilidade em decorrência da exposição do solo, 0 que demonstra que o NDRE obteve uma maior qualidade no processo para os 45 DAS.

Palavras-chave: Sensoriamento remoto; agricultura de precisão; OptRx. 
Evaluation of the quality of the vegetation indexes (NDRE and NDVI) for the peanut culture, using control charts

\begin{abstract}
Peanuts are considered to be one of the most important legumes,not only for its economic expression but also nutritionally. Remote sensing techniques appear as a tool with high potential, to assist in the development of peanut culture. In view of this, the objective was to assess which vegetation index has the best quality for monitoring peanut culture. The experiment was carried out in a commercial farm located in Dumont in the State of São Paulo. The experimental design was based on the premises of the Statistical Quality Control (CEQ) (Montgomery, 2009) containing 30 sample points with 30 x $30 \mathrm{~m}$ mesh. The evaluations were carried out during the development of the culture at 45, 65, 75 and 85 days after sowing (DAS). The NDVI and NDRE vegetation indexes (IVs) were evaluated with the help of the GreenSeeker and OptRX canopy sensors. The analysis of the variability of reflectance and IVs of the peanut culture was carried out by means of individual value control charts. The grouping of data observed at 45 DAS, showed greater amplitude of variation in NDVI. In 75 and 85 days after sowing (DAS), the grouping of values for the NDVI can be seen by the proximity of the points in relation to the average, in the individual values chart, that is, there was low variability in the data acquisition. It was observed that the NDRE index showed the highest quality of the process as it has less variability. Another factor found was that the reflectance of the soil did not influence the quality of the process, as happened with the NDVI, which had a high variability due to the exposure of the soil, which demonstrates that the NDRE obtained a higher quality in the process for the 45 DAS.
\end{abstract}

Keywords: Remote sensing; precision agriculture; OptRx.

\title{
Evaluación de la calidad de los índices de vegetación (NDRE y NDVI) para el cultivo de maní, utilizando gráficos de control
}

\section{RESUMEN}

Los cacahuetes se consideran una de las legumbres más importantes, no solo por su expresión económica sino también nutricionalmente. Las técnicas de teledetección aparecen como una herramienta con alto potencial para ayudar en el desarrollo del cultivo de maní. En vista de esto, el objetivo era evaluar qué índice de vegetación tiene la mejor calidad para monitorear el cultivo de maní. El experimento se llevó a cabo en una granja comercial ubicada en Dumont en el estado de São Paulo. El diseño experimental se basó en las premisas del Control Estadístico de Calidad (CEQ) (Montgomery, 2009) que contiene 30 puntos de muestra con una malla de $30 \times 30 \mathrm{~m}$. Las evaluaciones se llevaron a cabo durante el desarrollo del cultivo a los 45, 65, 75 y 85 días después de la siembra (DAS). Los índices de vegetación (IV) NDVI y NDRE se evaluaron con la ayuda de los sensores de cubierta GreenSeeker y OptRX. El análisis de la variabilidad de la reflectancia y las IV del cultivo de maní se realizó mediante gráficos de control de valores individuales. La agrupación de datos observados a 45 DAS mostró una mayor amplitud de variación en NDVI. En 75 y 85 días después de la siembra (DAS), la agrupación de valores para el NDVI se puede ver por la proximidad de los puntos en relación con el promedio, es decir, en la tabla de valores individuales, es decir, había poca variabilidad en la adquisición de datos. Se observó que el índice NDRE mostró la más alta calidad del proceso ya que tiene menos variabilidad. Otro factor encontrado fue que la reflectancia del suelo no influyó en la calidad del proceso, como sucedió con el NDVI, que tenía una alta variabilidad debido a la exposición del suelo, lo que demuestra que el NDRE obtuvo una mayor calidad en el proceso para el 45 DAS.

Palabras clave: Detección remota; Agricultura de precisión; OptRx.

\section{Introdução}

sensoriamento remoto vem ganhando cada vez mais espaço nas ciências agrárias pelo fato de ter diversas aplicações nos estudos 
agronômicos. Além do estudo com base no comportamento espectral da vegetação, essa ciência permite realizar estimativas de biomassa, previsões de produtividade entre outras aplicações.

Tratando-se da cultura do amendoim, cultivada em grande maioria no Estado de São Paulo, o Sensoriamento Remoto surge com uma nova ferramenta que tem grande possibilidade de solucionar os desafios diários que os produtores enfrentam. Dentre as vantagens da adoção dessa ciência, destaca-se a identificação de estresses e suas causas e ainda, pode, dentre outros aspectos, auxiliar na tomada de decisão sobre o momento ideal da colheita, buscando maximizar a produção e reduzir as perdas na operação (Almeida et al., 2019).

A interação da radiação com 0 alvo torna possível que 0 sensoriamento remoto adquira diversas respostas com rapidez e precisão (Santos, 2019). A radiação que incide sobre o alvo pode ser proveniente do próprio sensor utilizado, sendo esse considerado como sensor ativo, ou da luz solar, sendo esse tipo de sensor conhecido como sensores passivos, que são sensores embarcados em satélites e câmeras multiespectrais (Steinvall et al., 2013; Molin et al., 2015).

Diante do exposto, objetivou-se avaliar o monitoramento dos dados obtidos a partir de um sensor terrestre (OptRx) e verificar qual índice de vegetação (NDRE e NDVI) possuem melhor qualidade para a cultura do amendoim, utilizando cartas de controle.

\section{Material e métodos}

O experimento foi realizado em Fazenda comercial localizada em Dumont no Estado de São Paulo, em uma área de rotação com cana-deaçúcar. O solo da área experimental possui textura argilosa sendo classificado como Latossolo Vermelho de acordo com Embrapa (2013). Quanto ao clima desta região é Aw, isto é, tropical com inverno seco, de acordo com a classificação climática de Köppen (Alvares et al., 2013). Os dados pluviométricos da área experimental são apresentados na Figura 1, 
sendo que o período de condução do experimento foi realizado de outubro de 2016 a fevereiro de 2017

O delineamento experimental foi baseado nas premissas do Controle Estatístico de Qualidade (CEQ) (Montgomery, 2009) contendo 30 pontos amostrais com malha $30 \times 30 \mathrm{~m}$. As avaliações foram realizadas durante $o$ desenvolvimento da cultura aos 45, 65, 75 e 85 dias após a semeadura (DAS). Foram avaliados os índices de vegetação (IVs) NDVI e NDRE, com o auxílio dos sensores de dossel GreenSeeker e OptRX.

O OptRX é um sensor óptico ativo da marca Ag Leader, modelo ACS430, que faz 5 leituras por segundo com frequência de leitura de $5 \mathrm{~Hz}$, a altura ideal de trabalho recomendada é de 0,60 e 0,70 $\mathrm{m}$ do dossel, com faixa de leitura imageada $(0,36$ a $0,42 \mathrm{~m}) 33$ de $60 \%$ da altura de leitura a qual foi preservada neste estudo. Foi utilizado dois sensores embarcados em uma motocicleta (Figura 1).

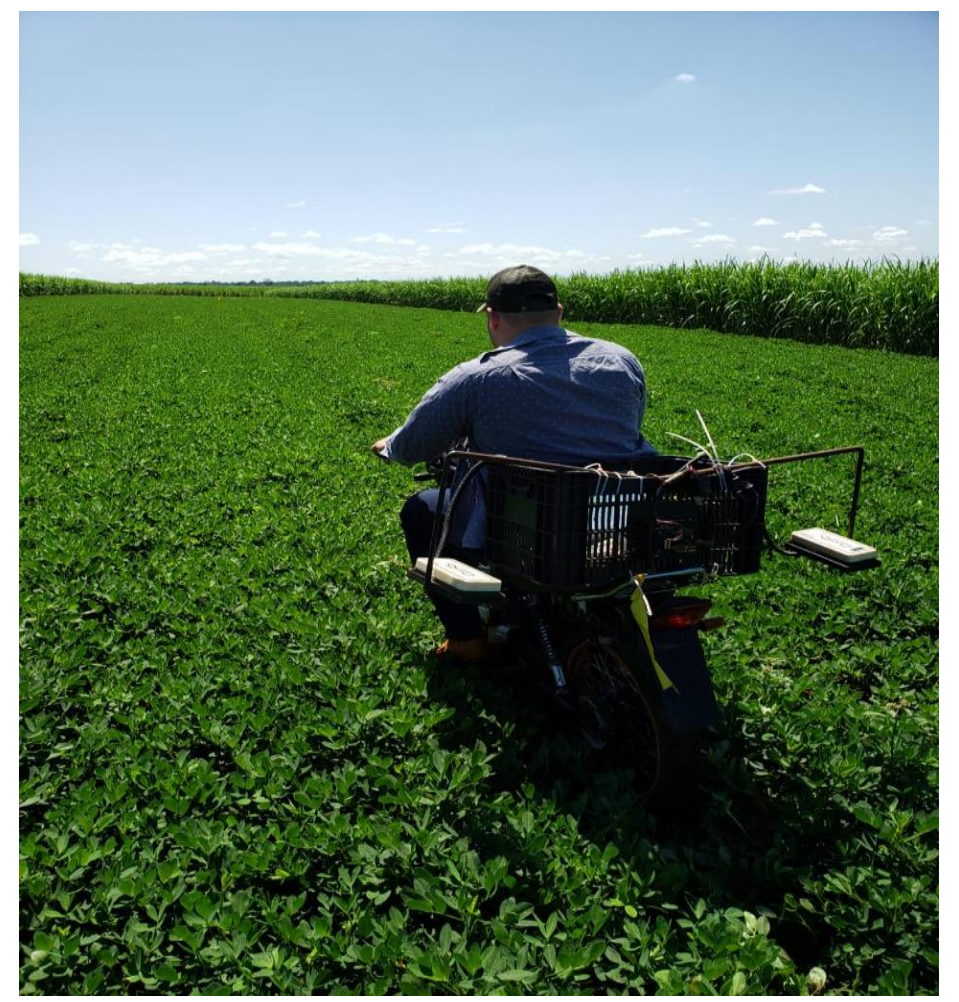

Figura 1. Sensor proximal OptRx embarcado em uma motocicleta.

O sensor emite uma luz própria e mede a reflectância de algumas ondas eletromagnéticas específicas, e essa luz medida se correlaciona com 
o teor de clorofila e a biomassa, permitindo ao sensor obter o índice de vegetação. Com esse sensor é possível obter os índices de vegetação NDVI e NDRE de acordo com a Tabela 1.

Tabela 1. Índices de vegetação.

Índice de vegetação Cálculo do índice

NDVI

$\mathrm{NDVI}=\frac{\text { Infravermelho próximo }- \text { Vermelho }}{\text { Infravermelho próximo }+ \text { Vermelho }}$

NDRE

$\mathrm{NDRE}=\frac{\text { Infravermelho próximo }- \text { Red Edge }}{\text { Infravermelho próximo }+ \text { Red Edge }}$

Para aquisição dos IVs, foram utilizadas alturas de trabalho padrões para os dois sensores de 0,6 a 0,7 m preservando as coletas dos IVs sempre nos mesmos horários no período da manhã entre 8h00 às 12 h00. Cada ponto amostral foi composto por duas linhas com 3 metros de comprimento com $0,90 \mathrm{~m}$ de espaçamento entre linhas, perfazendo $5,4 \mathrm{~m}^{2}$ de área útil por ponto.

A análise da variabilidade da reflectância e dos IVs da cultura do amendoim foi realizada por meio de cartas de controle de valores individuais que é uma das ferramentas do CEP e que permite monitorar comportamento dos dados ao longo do tempo com o auxílio do programa Minitab ${ }^{\circledR}$ (Minitab, 2007).

As cartas de controles de valores individuais são compostas pela média aritmética da amostra e pelos limites de controle superior (LSC) e inferior (LIC), obtidos pela média e desvio-padrão dos valores analisados por parâmetro.

\section{Resultados e discussão}

O agrupamento dos dados observado aos 45 DAS, apresentou amplitude de variação maior no NDVI. A variabilidade observada pode estar relacionada ao início do estabelecimento do amendoim, pois, nos estádios 
iniciais a cultura do amendoim apresenta dossel em desenvolvimento o que vai influenciar em maior reflectância do solo. Aos 65 DAS notou-se uma ligeira diferença na variabilidade do processo em relação aos 75 e 85 DAS.
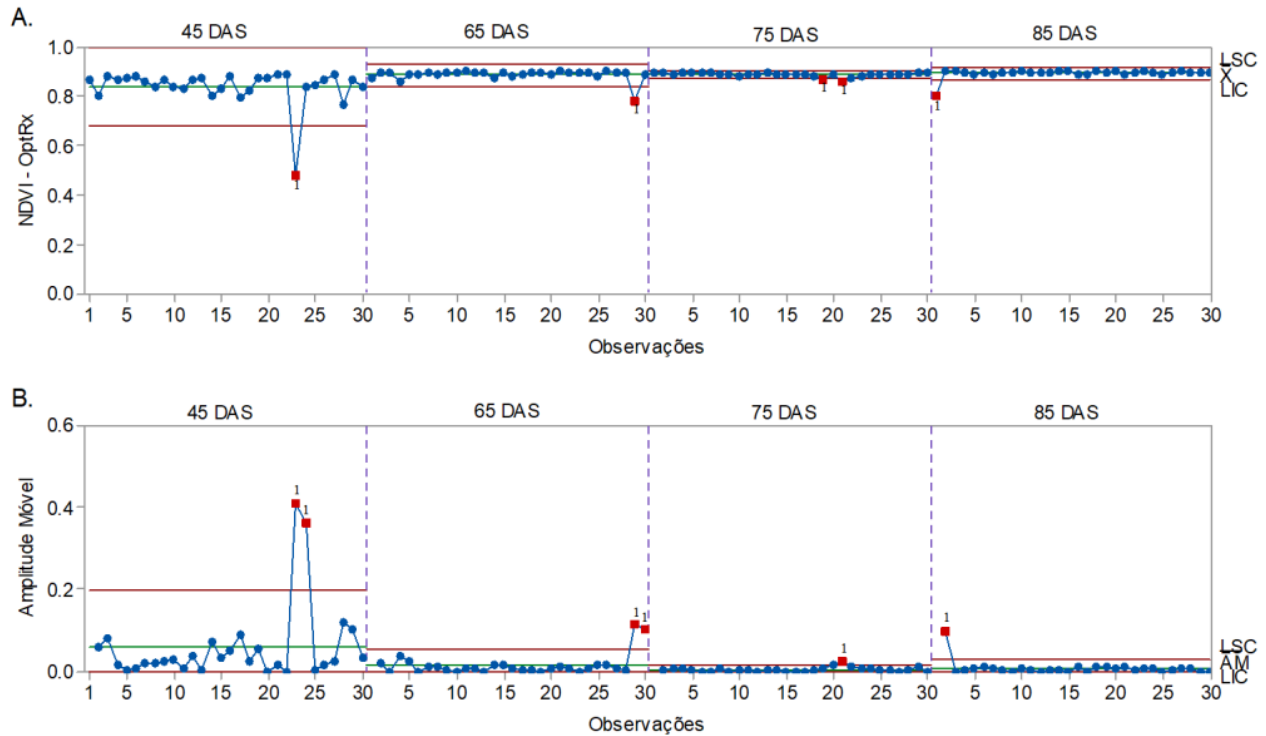

Figura 2. Análise da variabilidade dos índices de vegetação do NDVI utilizando o sensor proximal OptRx por meio das cartas de controle individual e amplitude móvel ao longo do tempo.

Em 75 e 85 dias após a semeadura (DAS), o agrupamento dos valores para o NDVI pode ser constatado pela proximidade dos pontos em relação à média, na carta de valores individuais (Figura 2), ou seja, houve baixa variabilidade da aquisição dos dados. Observando ainda esse processo é possível dizer que existe grande chances de o NDVI estar saturado devido ao mesmo comportamento demonstrado na carta de controle para 75 e 85 DAS. Segundo Baret e Guyot, (1991) esses resultados podem acontecer devido o NDVI apresentar como limitante a saturação na onda eletromagnético vermelha, em virtude de uma alta da alta assimilação dos pigmentos da clorofila, ocasionando a saturação do NDVI.

Foi observado que o índice NDRE apresentou maior qualidade do processo por ter menor variabilidade. Outro fator constatado, foi que o reflectância do solo não influenciou não qualidade do processo como aconteceu com o NDVI, que teve uma alta variabilidade em decorrencia da exposição do solo, o que demonstra que o NDRE obteve uma maior 
qualidade no processo para os 45 DAS. Amaral et al. (2015) e Taubinger et al. (2012) estudando a cultura da cana-de-açucar, observaram que o NDRE apresentou menos influência do dossel da planta e que foi mais eficiente para predizer a biomassa do que o NDVI.
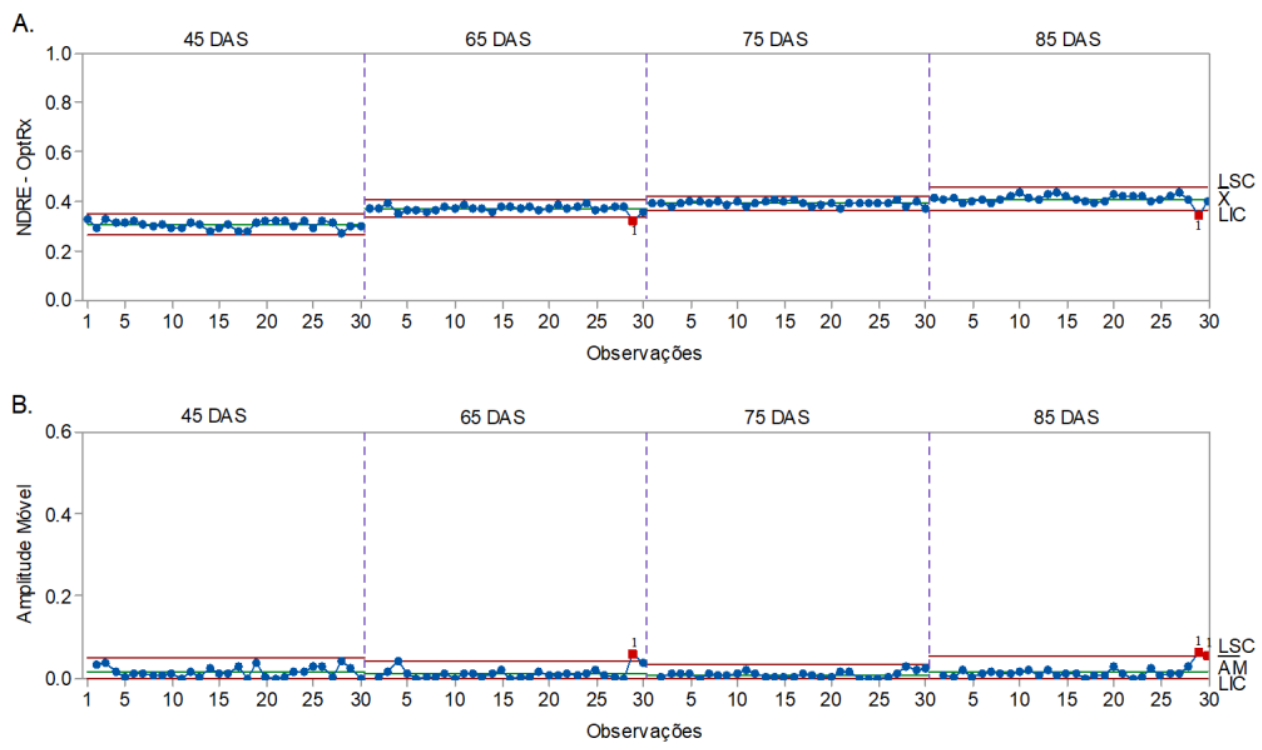

Figura 3. Análise da variabilidade do índice de vegetação do NDRE utilizando o sensor proximal OptRx por meio das cartas de controle individual e amplitude móvel ao longo do tempo.

Miller et al. (2018) Utilizando um sensor terrestre com o índice de vegetação NDRE avaliaram vários estádios de desenvolvimento da cultura da soja no período de 2015 e 2016 e os autores constataram que o NDRE pode ser usado em condições com maior biomassa do dossel vegetativo, o que se aplica no amendoim tendo em vista que a cultura tem um crescimento de biomassa considerável. Também foi possível observar nas cartas de controle em que o NDRE apresentou maior resistência de suas leituras a saturação do que o NDVI, sendo visível o crescimento do índice ao logo do tempo.

\section{Conclusões}

Para o monitoramento temporal da cultura por meio das cartas de controle, o NDRE obteve as melhores qualidades de leitura ao longo do processo comparado ao NDVI. 


\section{Referências}

ALVARES, C. A.; STAPE, J. L.; SENTELHAS, P. C.; GONÇALVES, J. L. M.; SPAROVEK, G. Köppen's climate classification map for Brazil. Meteorologische Zeitschrift, v. 22, p. 711-728, 2013.

AMARAL, L. R.; MOLIN, J. P.; PORTZ, G.; FINAZZI, F. B.; CORTINOVE, L. Comparison of crop canopy reflectance sensors used to identify sugarcane biomass and nitrogen status. Precision Agriculture, v. 16, p. 15-28, 2015.

BARET, F.; GUYOT, G. Potentials and limits of vegetation indices for LAI and APAR assessment. Remote Sensing of Environment, v. 35, p. 161-173, 1991.

EMBRAPA - Empresa Brasileira de Pesquisa Agropecuária. Sistema Brasileiro de Classificação de Solos, 3rd ed. Rev. ampl.; Brasília: EMBRAPA, 353p., 2013.

MILLER, J. J., SCHEPERS, J. S., SHAPIRO, C. A., ARNESON, N. J., ESKRIDGE, K. M., OLIVEIRA, M. C. et al. (2018). Characterizing soybean vigor and productivity using multiple crop canopy sensor readings. Field Crops Research, v. 216, v. 22-31. doi:10.1016/j.fcr.2017.11.006

MINITAB. MINITAB Release 16: Meet MINITAB 16. MINITAB StatGuide; MINITAB Help. [S.I.]: Minitab., 2007.

MOLIN, J. P., AMARAL, L. R., COLAÇO, A. F. (2015). Agricultura de precisão. 1a ed. São Paulo: Oficina de Textos.

MONTGOMERY, D. C. Introduction to Statistical Quality Control, 6th ed.; Arizona State University: John Wiley \& Sons, Inc., 754 p., 2009.

STEINVALL, O., CHEVALIER, M. E. T., GUSTAFSSON, O. (2013). Active and passive short-wave infrared and near-infrared imaging for horizontal and slant paths close to ground. Applied Optics, v. 52, n.20, p. 4763-4778, 2013.

TAUBINGER, L.; AMARAL, L. R.; MOLIN, J. P. Vegetation indices from active crop canopy sensor and their potential interference factors on sugarcane. p. 13. In: 11th INTERNATIONAL CONFERENCE ON PRECISION AGRICULTURE. Proceedings... Indianapolis: USA, p.13, 2012. 